\title{
Ejes para delinear un pensamiento crítico latinoamericano
}

\author{
Diego J. Duquelsky Gómez ${ }^{1}$ \\ Universidad de Buenos Aires \\ Universidad de José C. Paz - Argentina
}

Revista Derechos en Acción ISSN 2525-1678/ e-ISSN 2525-1686

Año 5/No 14, Verano 2019-2020 (21 diciembre a 20 marzo), 929-950

DOI: https://doi.org/10.24215/25251678e383

ORCID: https://orcid.org/0000-0003-3222-7544

\section{Presentación}

El presente trabajo es un paso más de un camino, siempre inacabado, que comenzó como una necesidad docente. Cuando en los cursos de teoría general, introducción o filosofía del derecho, terminaba de presentar los paradigmas tradicionales -iusnaturalismo y positivismo- me resultaba complejo explicar en pocas clases los distintos senderos en que se había desarrollado el pensamiento jurídico a partir de mediados del siglo XX.

Así comencé a elaborar una suerte de matriz de análisis que, cuatrimestre tras cuatrimestre, fue incorporando nuevas categorías, hasta alcanzar diez dicotomías que permitieran distinguir dos grandes perspectivas postpositivistas, a las que llamé liberales y críticas: a) modernos vs. posmodernos; b) racionalistas vs. irracionalistas; c) ilustrados vs. filosofía de la sospecha; d) constructivismo vs. deconstrucción; e) neocontractualismo vs. conflictivismo; f) lo individual vs. lo colectivo; g) universalismo vs. multiculturalismo; h) integridad vs. indeterminación;

1 Abogado, docente de la Universidad de Buenos Aires, Universidad Internacional de Andalucía y Universidad Nacional de José C. Paz. 
i) activismo tolerable vs. activismo indispensable; y j) fe vs. desconfianza en los juristas.

Esas mismas ideas, que comenzaron siendo un simple material didáctico de uso personal, han sido reelaboradas más de una vez y utilizadas con distintos propósitos. Por ejemplo para debatir con autores como Ferrajoli o Squella, o para poner en cuestión los presupuestos epistemológicos del pensamiento jurídico. $^{2}$

Pienso que vale la pena retomar nuevamente ese esquema de trabajo, a la hora de responder a la convocatoria celebrada para esta publicación, aunque se trate de una clasificación sesgada, arbitraria como todas, y pueda pecar de excesivamente simplista e imprecisa. Son dos "tipos ideales", con rótulos que -además- adolecen ambos de vaguedad combinatoria. Difícilmente encontremos autores o escuelas que adscriban simultáneamente a todos y cada uno de los criterios clasificatorios y, además, posiblemente deberemos admitir como parte del mismo grupo a pensadores con tesis incompatibles entre sí.

Sin embargo, más allá de sus falencias, pensamos que es posible encontrar en ella algunos ejes para repensar el pensamiento jurídico crítico latinoamericano, como una forma de superar los límites de las teorías críticas de raíz exclusivamente eurocéntrica.

\section{Modernidad/posmodernidad: ¿cuándo, cómo y dónde?}

El primer punto que propongo analizar, tiene que ver con la perspectiva histórica desde donde cuestionar tanto al

\footnotetext{
2 En el primer caso, en el encuentro celebrado en la facultad de Derecho de la UBA en abril de 2018, organizado por la Asociación Argentina del Derecho mediante el trabajo "Es todavía crítico el positivismo de Ferrajoli" que formará parte de una futura publicación. En el segundo, mediante mi colaboración en un libro homenaje a Agustín Squella, también actualmente en prensa, titulada "Yo soy un jurista crítico, y don Agustín?". Finalmente, me refiero a los trabajos presentados en el "Il Congreso de Filosofía del Derecho del Mundo Latino", celebrado en Río de Janeiro en julio de 2018 y en el "Congreso Nacional de Filosofía" Ilevado a cabo en la Universidad Nacional de Lanús, en abril de 2019.
} 
formalismo normativista como a las teorías post-positivistas de matriz liberal.

El positivismo puede ser visto como el resultado de un proceso que arranca en los siglos XVII y XVIII, que fue priorizando progresivamente la racionalidad científica por sobre todas las demás. Una racionalidad científica que, al momento de unirse al capitalismo, se torna fundamentalmente racionalidad productiva.

Una parte importante de la crítica al positivismo puede ser leída también como denuncia frente a las "promesas inclumplidas" de la modernidad. Y en esa línea se desarrollan trabajos que van desde Bobbio a Ferrajoli, pasando por Habemas, entre tantos otros.

La pregunta que cabe hacerse desde América Latina es el modo en que la modernidad nos ha sido impuesta y las posibilidades de "rehacer" algo que nunca fue. Por eso muchos proponemos pensar la crítica jurídica radical desde una perspectiva que, sólo a falta de un nombre mejor, podríamos llamar posmoderna, lo que requiere importantes precisiones terminológicas.

Existe un modo muy difundido de usar el término posmoderno al que Boaventura de Sousa Santos ${ }^{3}$ denomina "celebratorio". Desde esta perspectiva, advertir la falta de soluciones modernas para los problemas de la modernidad, no es en sí mismo un problema sino su solución. El error fue pensar que eran realizables esas promesas falsas e ilusorias. Para muchos posmodernos, ahora que sabemos la verdad, podremos finalmente reconciliarnos con la sociedad en la que vivimos, y celebrar lo que existe meramente tal cual es.

Por el contrario, el "posmodernismo de oposición" se presenta como alternativo tanto de las posturas modernas, como del posmodernismo celebratorio.

3 Santos, Boaventura de Sousa. Sociología jurídica crítica para un nuevo sentido común en el derecho. Bogotá: ILSA, 2009. Colección En clave de Sur.pag 42 y sgtes.

Otra terminología que puede resultar útil para referirse a esta etapa es la de "transmodernidad" propuesta por Enrique Dussel. Ver por ejemplo Dussel, E. "Transmodernidad e interculturalidad" 
El rasgo central del posmodernismo de oposición es la conciencia de su carácter provisional o transicional: no hay una condición posmoderna; hay un momento posmoderno. El propósito de Santos al designar este momento como posmoderno, sólo apunta a indicar la incapacidad de caracterizar adecuadamente esta etapa de transición desde nuestra propia mirada actual. Se trata de un momento entre un paradigma que es dominante todavía -que incluso es capaz de denunciar sus irremediables contradicciones, como hacen muchos teóricos liberales- y otro paradigma o paradigmas emergentes, de los que hasta el momento sólo tenemos algunos signos.

\section{Racionalismo/irracionalismo (o pluriracionalidades)}

El segundo par, íntimamente relacionado con la distinción entre modernos/posmodernos, es la dicotomía "racionalismo/ irracionalismo".

Según Boaventura de Sousa Santos, el proyecto emancipatorio de la modernidad se anclaba en tres dimensiones de la racionalidad y secularización de la vida colectiva: a) la moralpráctica del derecho moderno, b) la cognoscitivo-instrumental de la ciencia y técnica modernas y c) la estético-expresiva de las artes y de la literatura modernas.

Sin embargo, el pretendido equilibrio nunca fue tal, producto entre otros factores del desequilibrio interno del pilar de la emancipación: la racionalidad cognoscitivo-instrumental de la ciencia y de la técnica se desarrolló en detrimento de las demás, a las que terminó colonizando. En el campo jurídico, reduciendo la riquísima tradición de reflexión filosófica, sociológica y política sobre el derecho a mera ciencia dogmática.

De allí que, como señalábamos en el apartado precedente, una parte importante de pensadores que llamamos liberales y modernos, se caractericen por intentar revertir esa situación y recuperar la fe en la razón como herramienta no sólo para el conocimiento científico/tecnológico, sino como modo de guiar la acción, juzgar nuestras instituciones, fundamentar la democracia, etc. 
Es por todos conocido el nexo entre razón y modernidad toda vez que, como señala Habermas, más allá de ser una idea presente en el pensamiento aristotélico, "el concepto de razón práctica como capacidad subjetiva es una acuñación moderna"s.

Tampoco es el mismo el sentido con que autores como Nino, Rawls, Habermas o Alexy utilizan la idea de razón, en algunos casos de modo más semejante a la concepción kantiana en estado puro, otros reformulándola en el sentido de acción comunicativa, otros en términos de corrección procedimental o argumentativa, pero todos recuperando un sentido que -como veíamos en el apartado precedente- había sido censurado por el positivismo lógico.

Y así como era compleja la utilización del término "posmoderno", otro tanto acontece con la expresión "irracionalista", cuya carga emotiva negativa sigue siendo fuerte y difícilmente sea aceptada de buen grado incluso por muchos juristas autodenominados críticos. Intentaremos clarificar el concepto.

En primer lugar, se cuestiona la idea de una racionalidad única. Pensar en un modo único de concebir la razón es una de las manifestaciones del "epistemicidio" provocado por el proceso colonizador.

Tres siglos antes de que el positivismo lógico desterrara de la esfera de lo cognoscible todo aquello que no fuera verificable empíricamente, el pensamiento occidental moderno trazó una línea divisoria (abismal en términos de Boaventura de Sousa Santos) $)^{5}$ condenando a magia o superstición toda forma de conocimiento que no respondiera a los cánones europeos. Las dificultades de desarrollar un pensamiento crítico radical vienen de la mano del hecho que el fin del colonialismo político no significó el fin del colonialismo epistemológico.

\footnotetext{
4 Habermas, J., Facticidad y validez, Trotta, Madrid, 1998 p. 63.

5 Ver Santos, Boaventura de Sousa, Descolonizar el saber, reinventar el poder, Ediciones Trilce, Extensión universitaria: Universidad de la República, Montevideo, 2010. CAPITULO 2
} 
Aunque con un sentido más restringido, también el movimiento de los Critical Legal Studies acepta la expresión "irracionalismo" como la característica de una de sus vertientes internas, dando cuenta de cómo para un importante grupo de miembros del movimiento, la voluntad de desestabilización prima por sobre la voluntad de formar una escuela sobre premisas coherentes y una jerarquía interna ${ }^{6}$.

Como explica César Rodríguez, para los críticos posmodernos "todas las jerarquías conceptuales que privilegian un polo del discurso por sobre otro son características de la racional moderna, cuya médula consiste justamente en construir categorías conceptuales opuestas ( $v$. gr. sujeto-objeto) para luego favorecer una de ellas sobre la otra ( $v$. gr. sujeto/objeto). El resultado de esata práctica es la tiranía del orden conceptual existente que, gracias a que reclama estar fundado en la razón, parece natural, necesario."

\section{Ilustración/Filosofía de la sospecha: sobran motivos para desconfiar}

Otra característica que comparten muchos de los pensadores post-positivistas liberales, modernos, racionalistas es su reivindicación del pensamiento ilustrado, en particular en la figura de Kant.

Ese rasgo no es propio tan solo de los pensadores alemanes, como en el caso de Habermas y sus discípulos. Llega a América Latina, como en el caso de Nino e incluso en el mundo anglosajón, paradigmáticamente en la obra de John Ralws. Este último, un caso especialmente curioso, porque rompe con el predominio del utilitarismo y la filosofía analítica en el campo

\footnotetext{
6 KENNEDY, Duncan, Notas sobre la historia de CLS en los Estados Unidos, en Doxa N ${ }^{\circ} 11$, 1992, p. 286

7 RODRIGUEZ, Cesar, Una crítica contra los dogmas de la coherencia del derecho y la neutralidad de los jueces, Los estudios críticos del derecho y la teoría de la decisión judicial, Estudio Preliminar en KENNEDY, Duncan, Libertad y restricción en la decisión judicial, Siglo del Hombre Editores, Bogotá, 1999, p. 51.
} 
de la filosofía jurídica, moral y política anglosajona que había durado más de un siglo.

Para continuar con nuestras dicotomías, propongo poner el énfasis en la influencia central para el desarrollo de un pensamiento crítico de otra perspectiva, la denominada "filosofía de la sospecha".

La expresión "maestros de la sospecha", fue usada por primera vez en 1965 por Paul Ricoeur para referirse a la tríada de pensadores integrada por Karl Marx, Friedrich Nietzsche y Sigmund Freud. Una expresión que luego se difundió para hacer referencia a esta suerte de corriente o "escuela" integrada por autores que -como admite el propio Ricoeur- aunque quizás se excluyen entre sí en muchos aspectos, comparten una perspectiva, punto de vista o actitud: la desconfianza.

Nos dice Ricoeur: "Si nos remontamos a su intención común, encontramos alli la decisión de considerar en primer lugar la conciencia en su conjunto como conciencia "falsa". Por ahi retoman, cada uno en un registro diferente, el problema de la duda cartesiana, para llevarlo al corazón mismo de la fortaleza cartesiana. El filósofo formado en la escuela de Descartes sabe que las cosas son dudosas, que no son tales como aparecen; pero no duda de que la conciencia sea tal como se aparece a si misma; en ella, sentido y conciencia del sentido coinciden; desde Marx, Nietzsche y Freud, lo dudamos. Después de la duda sobre la cosa, entramos en la duda sobre la conciencia. ${ }^{\circledR}$

Obviamente, desde el punto de vista histórico y geográfico, se trata de pensadores modernos. Sin embargo, su ideas vienen a romper muchos de los presupuestos de la racionalidad occidental inaugurada por el iluminismo, fundamentalmente a partir de cuestionar al sujeto, su autonomía y su racionalidad:

\footnotetext{
8 "Esto es aún más cierto, sin duda, en la escuela de la sospecha que en la de la reminiscencia. La dominan tres maestros que aparentemente se excluyen entre sí: Marx, Nietzsche y Freud", Ricoeur, Paul, FREUD: UNA INTERPRETACIÓN DE LA CULTURA, siglo XXI, México, 1970, p. 32.
}

9 Ricoeur, op. cit., p. 33 
Nietzsche, con su modo de abordar la relación entre el poder y el saber, Marx mediante su concepto de ideología y Freud, a partir de la noción de inconsciente, ponen en crisis la posibilidad de pensar el mundo exclusivamente en términos racionales. Y si en la Europa de los siglos XIX y XX había motivos para sospechar de la razón moderna, el contexto latinoamericano, atravesado en forma adicional por el componente colonial, hace que sobren motivos para una actitud de desconfiada.

\section{Constructivismo/deconstrucción: lo ideal o lo oculto}

La siguiente de las dicotomías planteadas es casi una consecuencia lineal del planteo formulado: la oposición entre constructivismo y deconstrucción, como tarea primordial de las dos corrientes hasta aquí perfiladas.

Referirnos a "constuctivismo" implica apelar a una expresión marcadamente ambigua, aunque como suele pasar en la mayoría de los casos de ambigüedad, los distintos sentidos del término no son por completo independientes.

Mucho más que en el ámbito estrictamente jurídico, es en el campo de la filosofía moral y política donde el constructivismo aparece más directamente ligado al pensamiento post positivista. Así, tanto Habermas como Rawls y Nino son reconocidos como constructivistas.

Si tuviéramos que pensar en características comunes, o arriesgar una definición mínima, podríamos afirmar que los constructivistas no rechazan la posibilidad de afirmar la corrección de juicios morales universalmente válidos, pero lo hacen sin caer en posturas realistas en términos morales, ya que dichos juicios no serían juicios descriptivos estrictamente verdaderos o falsos, sino que se trataría de juicios racionalmente justificados.

Además, se trata de teorías procedimentales que parten de presupuestos artificialmente construidos de los cuales de derivarían racionalmente las soluciones "justas", como la "situación ideal de diálogo" habermasiana o la "posición originaria" de Rawls. 
Si la palabra clave para los liberales, modernos, racionalistas es el constructivismo, del otro lado la palabra clave será la deconstrucción y la deconstrucción no es otra cosa que buscar lo oculto. Lo que está detrás de las apariencias. Así como los postpositivistas modernos reprochan al positivismo el haber renunciado a usar la razón para el campo de la moral o la política, los críticos denunciarán todas las cosas que el positivismo normativista ha ocultado, ya que detrás de una prolija y ordenada "Teoría Pura del Derecho", o de unos racionales y lógicos "Sistemas Normativos", se escondía la ideología, las relaciones de poder, la dominación, la explotación. O sea, de lo que se trata es correr un poquito y mirar abajo de la alfombra, hablar de lo que no se habla.

Seguramente, el sentido en que con mayor fuerza las teorías críticas del derecho latinoamericanas han continuado la tradición del pensamiento marxista, ha sido presentar al derecho como un discurso ideológico ${ }^{10}$. Por eso una tarea central del pensamiento jurídico crítico ha consistido en mostrar los vínculos entre derecho e ideología, derecho e historia, derecho y poder. En develar el carácter político de la función judicial, el papel de la formación jurídica como mecanismo para general opacidad, para reproducir hegemonía, etc.

En una línea análoga, el movimiento de los Critical Legal Studies ha hecho de la deconstrucción una herramienta clave profundizando muchos de los señalamientos que a principios del siglo XX ya habían sido esbozados por el realismo jurídico norteamericano. Así como Holmes, Frank o Lewellyn son precursores en mostrar como elementos no estrictamente "jurídicos" influyen en la decisión judicial, los CLS incorporan la dimensión ideológico-política para realizar una tarea de "demolición" del material jurídico como sentencias, libros, manuales, etc.

\footnotetext{
10 En muchos aspectos claramente el pensamiento jurídico crítico se ha distanciado de las lecturas marxistas ortodoxas, ver al respecto CARCOVA, Carlos, Acerca de las relaciones entre derecho y marxismo, en Derecho, Política y Magistratura, Biblios, Buenos Aires, 1996.
} 


\section{Neocontractualismo/conflictivismo: ¿"quiénes" realmente acuerdan "qué"?}

La "democracia deliberativa" de Nino; "la comunidad ideal de diálogo" de Habermas; o la "posición originaria" de Rawls son de un modo u otro una suerte de "contratos sociales". De allí que puede afirmarse que otro rasgo común de los más relevantes teóricos post-positivistas de raíz liberal lo constituye su "neocontractualismo" más o menos explícito. Así como señalábamos la reivindicación del pensamiento ilustrado, también se retoma, como en Kant, Locke, o Hobbes, la vieja idea de contrato social.

Son autores que inventaron algunos mecanismos para, procedimentalmente, justificar la corrección o no de ciertos juicios morales, como los valores democráticos, en base a ponernos de acuerdo sobre ciertas cosas. La complejidad de esos mecanismos y su evidente artificialidad no los separan sin embargo de la raíz contractualista. También los iusnaturalistas de los siglos XVII y XVIII eran conscientes de que tanto el "estado de naturaleza" como el "contrato social" no eran hechos históricos, sino construcciones racionales. ${ }^{11}$

Frente a estas perspectivas, vale la pena restacar a aquellos autores a los que podríamos denominar genéricamente "conflictualistas". Ernesto Laclau, Chantal Mouffe o la propia Judith Buttler, son teóricos que no conciben al conflicto como una patología social y como un obstáculo para la democracia, sino todo lo contrario. Las relaciones sociales son relaciones conflictivas, el conflicto es ineliminable y el modelo de democracia deliberativa liberal, no hace otra cosa que ocultarlo o trasladarlo a la esfera de lo privado.

Recordando a Chantal Mouffe: El pensamiento liberal tiene que ser necesariamente ciego a lo político debido a que su individualismo le hace incapaz de entender la formación

\footnotetext{
11 Para un análisis profundo pero a la vez claro sobre la cuestión, el clásico texto de Bobbio, Norberto, El modelo iusnaturalista, en Sociedad y Estado en la filosofía moderna, Fondo de Cultura Económica, Mexico, 3ra. reimpresión 1996.
} 
de identidades colectivas. Sin embargo, lo político está desde el primer momento imbricado con formas de identificación colectivas por cuanto en este campo siempre estamos tratando de la formación de un "nosotros"por oposición a un "ellos". Lo político tiene que ver con el conflicto y el antagonismo. No tiene nada de extraño, pues, que el racionalismo liberal no sea capaz de aprehender su naturaleza, dado que el racionalismo requiere la negación misma de la inerradicabilidad del antagonismo. El liberalismo tiene que negar el antagonismo ya que, al situar en primer plano el momento ineludible de la decisión -en el sentido fuerte de tener que decidir en un terreno indecidible-, lo que el antagonismo pone de manifiesto es el límite mismo de todo consenso racional. ${ }^{12}$

\section{Individual/colectivo: repensando sujetos, pueblos, movimientos}

La crítica a los límites de la democracia liberal es determinada también por el modo en que se deconstruye el concepto de sujeto. No sólo el sujeto no es preexistente a la sociedad, al sistema político y particularmente al discurso jurídico, sino que será ese mismo discurso el que constituya su propia autoconcepción. Al mismo tiempo, la adopción de un pensamiento crítico implica el reconocimiento de subjetividades e identidades colectivas.

Es por eso que, posiblemente, sea éste uno de los puntos donde el pensamiento crítico latinoamericano tenga más que aportar -y consecuentemente también donde todavía haya más que explorar, pensar, crear.

Si, utilizando la terminología de Bachelard ${ }^{13}$, hiciéramos una suerte de psicoanálisis de la razón jurídica latinoamericana, y como parte del colonialismo cultural al que hacíamos

\footnotetext{
12 MOUFFE, Chantall: "Política agonística en un mundo multipolar". Documentos CIDOB. Dinámicas interculturales, Nro 15, Barcelona, 2010, p. 6.

13 BACHELARD, Gastón, La formación del espíritu científico, Siglo XXI, Buenos Aires, 1972.
} 
referencia previamente, veremos que hemos heredado algunos traumas ajenos.

Es muy atendible que los autores europeos, que sufrieron regímenes totalitarios que accedieron al poder con un fuerte apoyo popular y que en nombre del pueblo han avasallado los derechos de las minorías, tengan particular recelo a los avances antidemocráticos de movimientos populares.

Pero, como hemos sostenido en más de una ocasión, no debemos perder de vista que las nociones de mayorías y minorías no pueden asociarse directamente con situaciones de mayor o menor poder real. En nuestras sociedades existen minorías privilegiadas y poderosas y enormes mayorías que representan a los sectores más débiles y vulnerables.

Nuestras dictaduras han sido siempre dictaduras de las minorías, que en nombre de la república, la democracia y la libertad han sistemáticamente violado los derechos que decían defender. Como contracara, incluso desde el pensamiento de izquierda latinoamericano, prácticamente hasta la aparición de la obra de Laclau $^{14}$, se aceptó con liviandad la carga emotiva necesariamente negativa o peyorativa del término populismo.

Al mismo tiempo, América Latina ha sido fuente de una nueva forma de concebir los sujetos colectivos, que si bien ha tenido importantes desarrollos en el campo de las ciencias sociales, no ha penetrado lo suficiente en el campo de la filosofía del derecho.

La crisis del modelo neoliberal en Latinoamérica, a fines de los años noventa, no sólo implicó simultáneamente una crisis política sin precedentes, sino que resignificó el papel de los Nuevos Movimientos Sociales. En el caso de Bolivia, por ejemplo, el proceso que desemboca en la aprobación de la constitución de 2009 hubiera sido imposible mediante un sistema de representación político partidario o gremial tradicional. En ese sentido, el ejemplo boliviano no debería ser menospreciado a

14 Ver LACLAU, Ernesto, La razón populista, Fondo de Cultura Económica, Buenos Aires, 2005 
la hora de reformular el nexo entre la ciudadanía y la determinación de sus modos de gobierno. ${ }^{15}$

\section{Universalismo/particularismo: la falsa dicotomía entre multiculturalismo y derechos humanos}

Tratando de simplificar algunos de los términos del debate, es posible pensar que hay una línea argumental común que hace desembocar a las características previas de las concepciones liberales del lado del universalismo y, por el contrario, a las teorías críticas cercanas al reconocimiento del multiculturalismo.

Ello es así en la medida que, si defendemos algún tipo de pretensión cognitiva en materia de razonamiento moral, si establecemos ciertos procedimientos o mecanismos que aíslan las circunstancias coyunturales, si realizamos ciertas construcciones intelectuales, etc., lo es, precisamente, para garantizar el carácter universal de nuestros resultados.

Para los autores liberales la cuestión es bastante sencilla. Basta con presentar teorías de "corto alcance", que se contenten con sostener posiciones que pretenden ser compatibles con distintas concepciones del bien. En ese sentido es paradigmática la concepción de Rawls, que como sabemos no pretende más que ser una "teoría política" de la justicia, con la búsqueda de un consenso traslapado o superpuesto. ${ }^{16}$

Esas posturas van acompañadas de una cierta concepción -también limitada- de cosmopolitismo. Aspiran, simultáneamente, trascender el Estado Nación y al mismo tiempo, ser compatibles con distintas culturas, aunque claro, aquellas culturas que ellos mismos consideran "razonables".

\footnotetext{
15 Ver CHAPLIN, Ann, "Movimientos sociales en Bolivia: de la fuerza al poder"; Oxford University Press and Community Development Journal, 2010 [En línea].

16 Rawls, J. "La Justicia como equidad: política no metafísica", Mazzuca, S. (trad.), Política. Revista de Estudios sobre el Estado y la Sociedad 1, 1996, 23-46. Y Rawls, J. El liberalismo político, Domènech, A. (trad.). Barcelona: Crítica, 1996
} 
Las corrientes críticas, en cambio, advierten la complejidad del debate en torno a los derechos humanos que pueden concebirse desde dos puntos de vista: como un localismo globalizado o como un cosmopolitismo subalterno e insurgente ${ }^{17}$. Por eso es necesario apartarse de las miradas eurocéntricas, aun aquellas autopercibidas como críticas y asumir que la única alternativa para superar la dicotomía universalismo/ particularismo es el intento de una reconstrucción genuinamente intercultural de los derechos humanos.

Dicha reconstrucción intercultural, según Boaventura de Sousa Santos, debería asentarse sobre algunos presupuestos que aquí sólo enunciaremos: a) conciencia de la incompletitud de todas las culturas, b) reconocimiento de la diversidad interna de todas las culturas; c) el respeto por los distintos tiempos para el diálogo intercultural; d) una agenda de temas planteada horizontalmente y no impuesta por cierta cultura y e) el derecho a ser iguales cuando la diferencia nos inferioriza y el derecho a ser diferentes cuando la igualdad pone en peligro la identidad. ${ }^{18}$

\section{Integridad/indeterminación: otra vez entre "nobles sueños" y "pesadillas"}

Así como Nino, Rawls o Habermas son autores post-positivistas que se destacan en el plano de la teoría política, otros como

\footnotetext{
17 Santos reconoce cuatro formas de globalización. Llama "localismo globalizado" al proceso por el cual un fenómeno local es globalizado con éxito (por ejemplo el uso de la lengua inglesa como lingua franca). Globalismo localizado, que consiste en el impacto específico de las prácticas transnacionales en las condiciones locales (por ejemplo, el uso turístico de tesoros históricos, desforestación y deterioro de recursos naturales para pagar deuda, etc), Denomina "cosmopolitismo" a prácticas globales contrahegemónicas (por ejemplo organizaciones sindicales sur/sur, foros antiglobalización) y finalmente "herencia común de la humanidad" a los asuntos que afectan inexorablemente a toda la humanidad (problema nuclear, sida, etc.). Ver Santos, Boaventura de S., La globalización del derecho, Universidad Nacional de Colombia, Bogotá, 1999, pp. 48 y sgtes.

18 Para un desarrollo más amplio de estos puntos ver Santos, Boaventura de Sousa, DescoIonizar el saber, reinventar el poder, Ediciones Trilce, Extensión universitaria: Universidad de la República, Montevideo, 2010. CAPITULO 3.
} 
Dworkin, Alexy, Zagrebelsky o Atienza se caracterizarán por sus desarrollos en el plano de la argumentación e interpretación jurídica.

Siguiendo con las simplificaciones, podríamos encontrar algunos rasgos en común a todos estos autores.

El primero tiene que ver con la constatación -en oposición a teorías de las normas como la desarrollada por Hart- de que el orden jurídico no se encuentra integrado exclusivamente por normas, sino también por principios.

En segundo lugar, la incorporación de principios opera una doble modificación del rol del juez en relación con la visión formalista. Por un lado, que la sujeción del juez a la ley ya no es sujeción a la ley cualquiera sea su contenido, sino guiada por tales principios. Por otro, que la tarea de subsunción, es reemplazada por la idea de ponderación, en caso de eventual conflicto entre principios.

La tercera característica tiene que ver con la idea de integridad o coherencia del orden jurídico. Aun cuando estos principios puedan parecer contradictorios, realizando adecuadamente la compleja práctica argumentativa en la que consiste la labor jurídica, es posible arribar a una respuesta correcta. En algunos casos se tratará de aplicar adecuadamente una fórmula, como en el caso de Alexy, en otros como Dworkin una tarea hercúlea de búsqueda en la tradición jurídico política de la comunidad.

Con muchas variantes, además, y dicho con terrible liviandad, podríamos sostener que estos autores piensan en dicha actividad como un ejercicio de la razón práctica y por lo tanto, que también hay un vínculo entre la solución moral y jurídicamente correcta.

En sus distintas variantes, por el contrario, las teorías críticas confluyen al menos en dos niveles de análisis insoslayables para poner en cuestión estos presupuestos.

Por una parte, en sintonía con las críticas formuladas por los Critical Legal Studies -para muchos los continuadores del realismo jurídico norteamericano- se pone énfasis en el plano 
ideológico y legitimatorio del discurso judicial. Así, por ejemplo, la actividad del juez pensado por Dworkin no se desarrolla heroicamente tras la búsqueda del ideal democrático de la sociedad, sino que detrás de su discurso hay fuertes componentes políticos.

Uno de los puntos más interesantes para rescatar de los CLS, fundamentalmente en Kennedy, consiste en advertir que la tensión entre "libertad" y "restricción" en la actividad judicial no obedece a las cualidades de los materiales jurídicos, sino que es una sensación o experiencia vivida por el propio magistrado.

Frente a los mismos hechos y con las mismas normas un juez puede sentir que la norma es clara y tiene no mucho margen de creación y otro, con distinta formación, valores o creencias, considerar que se encuentra frente a un caso atípico y debe recurrir a otros principios y normas para resolver la cuestión. ${ }^{19}$

En segundo lugar, cabe destacar que en su búsqueda de la construcción de un saber multi y transdisciplinario, las teorías críticas han asumido como centrales los aportes de la semiótica y la lingüística, que torna casi ingenua la idea de pensar en un sentido único de los materiales jurídicos. Como dice Cárcova: "la bermenéutica, la teoría del discurso, la teoría de la comunicación, el constructivismo lingüístico, el fenómeno de la narratividad, han tenido un impacto trascendente en la teoría del derecho y en la filosofía jurídica y han comenzado a proveer insumos de nuevo tipo para reanimar la reflexión jusfilosófica, como no podía ser de otro modo, dado que la materia prima

\footnotetext{
19 Pensemos, por ejemplo, en los planteos sobre matrimonio igualitario. Durante años a la gran mayoría de los jueces le hubiera parecido que si dos personas del mismo sexo querían contraer matrimonio esto era imposible ya que la ley no daba lugar a ninguna duda, claramente hacía referencia a un hombre y una mujer. Otros jueces, sin embargo, frente a hechos idénticos, con la misma constitución y con la misma ley vigente han encontrado en el precepto legal una limitación que consideraban violentaba precepto de rango superior. Ver el fallo de la jueza Gabriela Seijas Expte. № 34292 /0 caratulado FREYRE ALEJANDRO CONTRA GCBA SOBRE AMPARO (Art. 14 CCABA), que tramitó en el Juzgado de Primera Instancia en lo Contencioso Admnistrativo y Tributario de la Ciudad Autónoma de Buenos Aires.
} 
fundamental que constituye el objeto al cual ellas refieren es el lenguaje y más genéricamente, el sentido y la comunicación"20.

Nótese que cuando hablamos de interpretación no nos limitamos al plano meramente normativo, sino que aparece otro plano que también constituye un obstáculo complejísimo para entender el rol de la jurisdicción: la interpretación de las circunstancias fácticas.

Los aportes de la teoría literaria y la narratología ${ }^{21}$ resultan claves para entender que el proceso judicial no deja -ni puede dejar de ser- un relato. Así, podremos estar atentos y develar los niveles de distanciamiento entre lo narrado y lo acontecido, ya que el discurso jurídico siempre se construye en forma parcial, fragmentada, seleccionada. ${ }^{22}$

\section{Activismo tolerable/activismo indispensable: las particularidades del contexto latinoamericano}

Como hemos sostenido en un trabajo reciente: "a partir del surgimiento de teorías de corte post-positivista y el abandono de la figura del juez como mecánico aplicador de la ley, del papel asignado a los principios y del carácter normativo de los textos constitucionales, se ha generado una doble preocupación: por un lado, el temor a que ante la falta de límites derivada de la vaguedad propia de la incorporación de principios, se produzca un aumento en el poder discrecional de los jueces que ponga en riesgo las garantías constitucionales de los ciudadanos. Por

20 CÁRCOVA, C., 2009: <<Hay una traducción correcta de las normas 》, Revista Electrónica del Instituto de Investigaciones Jurídicas y Sociales Ambrosio Lucas Gioja, Vol. 3, 2009, p. 35

21 Esto no implica que haya una identidad automática entre el movimiento "derecho y literatura" y el pensamiento jurídico crítico, pero no deja de ser cierto que muchos autores críticos forman parte del mismo. Ver Roggero, Jorge (comp.) Derecho y Literatura. Textos y contextos, Eudeba, Buenos Aires, 2015.

22 Un trabajo pionero en la materia es el clásico texto de Marí, E. Moi Pierre Rivière y el mito de la uniformidad semática de las ciencias jurídicas y sociales en V.V.A.A., El discurso jurídico. Perspectiva psicoanalítica y otros abordajes epistemológicos, Hachette, Buenos Aires, 1982. 
otro lado, que voluntad popular expresada a través de la representación politica, quede sometida a poder contramayoritario de los magistrados judiciales". ${ }^{23}$

La pregunta que cabe preguntarse, frente a este modelo restrictivo de actuación frente a antinomias y lagunas, es si hay chances reales, al menos en el contexto latinoamericano, de conseguir efectivamente su eliminación, o deberemos contentarnos con regodearnos desde la crítica externa a señalar su existencia, mientras personas de carne y hueso ven vulnerados sus derechos fundamentales.

Al mismo tiempo, podríamos cuestionar si el respeto de la constitución y la eliminación de antinomias es sólo deber de la justicia constitucional, o alcanza a todos los magistrados. ${ }^{24}$

Consciente de dichas limitaciones, Manuel Atienza acuña la expresión "activismo tolerable" advirtiendo que el umbral de aceptación de la intervención judicial en materia de eliminación o colmado de lagunas pueda no ser el mismo en sistemas jurídicos diversos: "puede entenderse que los jueces latinoamericanos sean más activos que los europeos (los italianos o los españoles) a la hora de reconocer (o de "crear») derechos sociales, simplemente porque si ellos no lo hacen, no cabe esperar que alguna otra instancia estatal pueda satisfacer la exigencia que se plantea cuando alguien pide que se le reconozca uno de esos derechos: a la subsistencia, a la salud, etc." (ATIENZA, 2011: 85)

Pensamos que desde una concepción crítica del derecho es posible dar un paso más, y señalar que muchas veces lo tolerable se torna imprescindible. Que en muchos casos -y no

\footnotetext{
23 Ver DUQUELSKY, Diego, 2018 "La falsa dicotomía entre garantismo y activismo judicial" en Doxa $\mathrm{N}^{0} 41$.

24 Obviamente esto también estará condicionado con el sistema de control de constitucionalidad vigente en los distintos ordenamientos. Por otra parte, si bien es cierto que excedería el objeto de este trabajo, centrado en la actividad jurisdiccional, cabe reconocer como un déficit en los desarrollos de las teorías críticas del derecho la escasa atención a los incumplimientos normativos por parte de otros órganos de gobierno como los poderes ejecutivos y legislativos.
} 
sólo en materia de derechos económicos, sociales y culturales, el único modo posible de hacer prevalecer "los derechos sobre los poderes" -para usar una expresión cara a la teoría de Ferrajoli- es mediante un "activismo garantista".

La idea de un "activismo garantista" pretende señalar que es posible que los jueces adopten una actitud creativa, antiformalista y comprometida con la efectivización de los derechos fundamentales sin que ello implique la vulneración de garantías constitucionales.

Si la legitimación del juez radica en la democracia sustancial y no en la política, pensar que la satisfacción de los derechos fundamentales sólo se logra mediante el reenvío a las autoridades legislativas, difícilmente ayude a alcanzar el objetivo.

Ser conscientes de ésto tiene implicancias no sólo en términos de exigibilidad de los derechos económicos, sociales y culturales, sino también en el campo de las libertades civiles de otros grupos desaventajados -pienso en casos como el matrimonio igualitario, la identidad de género, derechos reproductivos, entre otros- e inclusive en materia penal.

\section{Fe/desconfianza en los juristas: de nuevo sobre la función paradojal del derecho}

Entonces ¿podemos o no tener confianza en el derecho y los juristas para transformar la realidad?

Para las concepciones liberales, modernas, constructivistas, sin dudas la respuesta será positiva. Si somos capaces de recuperar los valores de la ilustración, convertir racionalmente al derecho como un modo de garantizar la libertad, la igualdad y la fraternidad, si conseguimos construir modelos que nos permitan conocer y distinguir lo correcto de lo incorrecto, si la democracia nos otorga herramientas participativas para que las normas sean el resultado de una deliberación amplia, si logramos que en el ejercicio de sus prácticas argumentativas los operadores jurídicos hagan uso del razonamiento práctico y 
arriben a soluciones justas, ¿no sería razonable tener fe en el derecho? Es más, nuestra obediencia a las normas estaría a un paso de encontrarse justificada no sólo en base a la coerción, sino también moralmente.

Desde el punto de vista crítico, como hemos visto, resultan insostenibles todos esos presupuestos ideales. Como señalábamos en un trabajo reciente ${ }^{25}$, no podemos olvidar el papel profundamente conservador que históricamente ha cumplido el discurso jurídico en América Latina, particularmente a través de vastos sectores del poder judicial -anclado en estructuras tendientes a reproducir las formas dominantes de control social-, reforzadas desde el ámbito académico-jurídico, con una visión preponderantemente formalista y acrítica. Consideraciones que podríamos hacer extensivas a otros ámbitos jurídicos, como el mundo académico.

¿No hay nada que se pueda hacer, entonces?

Pensamos que uno de los principales aportes de las teorías críticas del derecho en la Argentina ${ }^{26}$ fue abandonar la lectura lineal del marxismo de concebir al derecho simplemente como un elemento superestructural de reproducción de relaciones de dominación, para desarrollar la idea de "función paradojal". El papel del derecho dependerá de una relación de fuerzas que permite determinar que "en manos de los grupos dominantes constituye un mecanismo de preservación y de reconducción de sus intereses y finalidades; en manos de los grupos dominados, un mecanismo de defensa y contestación política". ${ }^{27}$

Para que ese uso emancipatorio y alternativo del derecho sea posible, se torna indispensable ser conscientes no sólo de las

\footnotetext{
25 DUQUELSKY, Diego, "La falsa dicotomía entre activismo y garantismo judicial”, op. cit.

26 A través de los trabajos de Enrique Marí, Carlos Cárcova, Alicia Ruiz, Ricardo Entelman, Lucía Aseff, Claudio Martyniuk, Jorge Douglas Price o Mario Portela entre tantos otros (ver por ejemplo AAVV Materiales para una Teoría Crítica del Derecho, Abeledo Perrot, Bs. As., 1991 o AAVV, Desde otra mirada, Eudeba, Buenos Aires, 2001.)

27 CARCOVA, Carlos M., Notas acerca de la teoría crítica del derecho, en AAVV, Desde otra mirada, Eudeba, Buenos Aires, 2001.
} 
limitaciones del pensamiento moderno liberal sino también de las propias teorías críticas de raíz eurocéntrica, en múltiples niveles. A modo de cierre de este trabajo, entonces, me centraré en aquellos tres obstáculos que puedo visualizar con más claridad.

En primer lugar, que los modos de dominación que atraviesan nuestras sociedades son particularmente complejos y es imposible abordarlos críticamente sin una mirada simultáneamente anticapitalista, anticolonialista y antipatriarcal. Ninguna de las tres perspectivas, tomada aisladamente, resulta suficientemente emancipatoria.

En segundo término, como bien señala Boaventura de Sousa Santos, las teorías críticas de raíz eurocéntricas se han concebido a sí mismas como un pensamiento de vanguardia, generado desde los países centrales con el objeto de liderar y guiar los reclamos de los movimientos de corte progresista alrededor del mundo.

Sin embargo, en lo que va del siglo XXI, las luchas sociales más transformadoras e innovadoras, se han generado desde el Sur, en contextos socio-político-culturales muy distintos a los pensados por los intelectuales de izquierda de la elite académica mundial.

Por eso, la idea que propicia Boaventura es priorizar lo que denomina teorías de retaguardia, es decir, teorías "que acompanen muy de cerca la labor transformadora de los movimientos sociales, cuestionándola, comparándola sincrónica y diacrónicamente, ampliando simbólicamente su dimensión mediante articulaciones, traducciones, alianzas con otros movimientos $" 28$.

Finalmente, y muy ligado a lo anterior, es imperioso tender puentes entre las teorías críticas y los operadores jurídicos de carne y hueso, que son actores cruciales a la hora de llevar a cabo las luchas por los derechos.

Es notable como una parte importante del pensamiento crítico latinoamericano del siglo $\mathrm{XX}$ renegó de lo local, del

28 SANTOS, BOAVENTURA DE SOUSA, Descolonizar el saber, reinventar el poder, Ediciones Trilce, Extensión universitaria: Universidad de la República, Montevideo, 2010. 
barro y se limitó a sofisticadas discusiones teóricas con autores europeos. La creciente profesionalización de la academia sigue siendo caldo de cultivo para ese tipo de prácticas, con sus criterios burocráticos de evaluación, exigencia de publicación en determinado modelo de revista científica, etc.

Así como Alicia Ruiz se refería a los magistrados como los "modernos brujos"29 y Cárcova pensaba en el lenguaje jurídico como uno de los grandes generadores de "opacidad del derecho $^{30}$ ", tenemos que ser lo suficientemente autocríticos para advertir que hemos desarrollado una terminología críptica y hemos dado por sentados una serie de conocimientos previos -conceptos, corrientes, autores, etc.-, que operan como barreas infranqueables para la mayoría de abogados, jueces, fiscales e incluso académicos de las disciplinas dogmáticas.

De allí el desafío de pensar en modos de construcción de un saber que logre discutir y disputar espacios en la elaboración de planes de estudios de grado, posgrado, escuelas judiciales, colegios de abogados y demás ámbitos desde los que contribuir a la formación del sentido común jurídico alternativo y emancipatorio, que merezca llamarse crítico.

29 Ruiz, Alicia E. C., "La ilusión de lo jurídico”, en Materiales para una teoría crítica del derecho, Bs. As., Abeledo-Perrot, 1991

30 Cárcova, Carlos M., La opacidad del derecho, Trotta, Madrid, 1998 